60-61| 2018

La culture dans l'enseignement du français langue étrangère: conceptions théoriques, programmes et manuels auX $\mathrm{XIX}^{\mathrm{e}}$ et $\mathrm{XX} \mathrm{X}^{\mathrm{e}}$ siècles

\title{
La dimension culturelle dans l'enseignement du FLE : la littérature du Moyen Âge dans les manuels espagnols (1920-1970)
}

The cultural dimension in the teaching of French as a foreign language:

medieval literature in Spanish textbooks (1920-1970)

Juan Francisco García Bascuñana

\section{OpenEdition}

Édition électronique

URL : https://journals.openedition.org/dhfles/5098

DOI : $10.4000 /$ dhfles.5098

ISSN : 2221-4038

Éditeur

Société Internationale pour l'Histoire du Français Langue Étrangère ou Seconde

Édition imprimée

Date de publication : 1 janvier 2018

Pagination : 123-137

ISSN : 0992-7654

\section{Référence électronique}

Juan Francisco García Bascuñana, « La dimension culturelle dans l'enseignement du FLE : la littérature du Moyen Âge dans les manuels espagnols (1920-1970) », Documents pour l'histoire du français langue étrangère ou seconde [En ligne], 60-61 | 2018, mis en ligne le 01 juin 2019, consulté le 29 mars 2023. URL : http://journals.openedition.org/dhfles/5098; DOI : https://doi.org/10.4000/dhfles. 5098

Ce document a été généré automatiquement le 29 mars 2023

Tous droits réservés 


\section{La dimension culturelle dans} l'enseignement du FLE : la littérature du Moyen Âge dans les
manuels espagnols (1920-1970)

The cultural dimension in the teaching of French as a foreign language: medieval literature in Spanish textbooks (1920-1970)

Juan Francisco García Bascuñana

\section{Introduction}

1 Nous nous proposons d'aborder ici un aspect qui nous semble spécialement pertinent et qui a été négligé par ceux qui se sont intéressés à la présence de la littérature dans l'enseignement du français en Espagne. Nous nous référons à l'absence des auteurs du Moyen Âge dans les manuels de français pour Espagnols. Ce qui a été justifié avec des arguments divers: d'une part, à cause des problèmes linguistiques que les textes médiévaux peuvent comporter pour des raisons évidentes, ce qui suppose une difficulté majeure pour un apprenant étranger; d'autre part, parce que la littérature du Moyen Âge a été découverte tardivement par les auteurs de manuels de français langue étrangère, épris qu'ils étaient des grands auteurs classiques en tant que modèles littéraires, langagiers et moraux. Pas même le " goût du Moyen Âge " qui s'impose en France au XIXe siècle n'aurait favorisé sa présence dans les manuels de français. Aucun auteur d'anthologies littéraires ou de manuels du XIX siècle destinés à l'enseignement $d u$ français en Espagne, à quelque exception près ${ }^{2}$, n'a inclus dans ses œuvres des textes littéraires médiévaux. Mais cette situation commence à changer clairement entre la fin de la Première Guerre mondiale et les années de la II $^{e}$ République espagnole, où un intérêt renouvelé pour le Moyen Âge a fait naître un engouement pour cette période, aussi bien dans les études universitaires que dans le secondaire. Une bonne partie des auteurs de manuels et des professeurs de français de l'époque seront avant tout, à cause 
de leur formation universitaire, des romanistes, avec toutes les conséquences pratiques que cela implique pour leur enseignement de la langue. Cette situation va perdurer d'ailleurs après la fin de la Guerre civile (1939) grâce à un intérêt renouvelé pour le Moyen Âge, suscité alors surtout pour des raisons purement idéologiques.

\section{La « redécouverte » du Moyen Âge}

2 Pendant les années 1920 et 1930 va naître en Espagne, comme nous venons de le dire, un intérêt renouvelé pour le Moyen Âge, une « redécouverte ", dirions-nous, après la première « découverte » au temps du Romantisme. Elle concernera aussi les auteurs de manuels pour l'enseignement du français langue étrangère. La raison de ce nouveau "goût du Moyen Âge " provient de la formation même des professeurs de français espagnols de l'époque, car une bonne partie d'entre eux réalise, dans les facultés des lettres où ils se forment, des études de philologie romane. Pour ces professeurs, enseigner le français devient l'occasion de s'approcher d'une littérature prestigieuse susceptible de conférer plus de valeur à leur enseignement, de sorte qu'ils puissent côtoyer les spécialistes les plus réputés. Surtout que cette nouvelle situation coïncide dans le temps avec le débat qui a lieu, dans des milieux comme l'Institución Libre de Enseñanza $a^{3}$ (Institution Libre d'Enseignement), à propos des vrais moyens et buts de l'enseignement des langues étrangères, auquel participent, entre autres des savants comme le philologue et historien Américo Castro ou le professeur de psychologie Rubén Landa (Roig 1999: 230-232). Même si la question est toujours posée à propos du véritable intérêt des textes médiévaux ou préclassiques pour des étudiants de français qui devaient acquérir surtout des compétences communicatives langagières. Mais l'ambiguïté subsiste alors et cela durera encore longtemps à propos de cet enseignement, et cela malgré les débats méthodologiques qui ont lieu à l'époque, concernant la place du français aussi bien dans le secondaire que dans les universités (Roig 1999 : 243-244). Le débat qui domine surtout au début des années 1930, tiraillé entre des positions contradictoires, va être imprégné d'un nouveau discours sur l'enseignement des langues étrangères, où tout en insistant sur l'importance des compétences langagières on va mettre l'accent sur leur dimension culturelle; c'est à partir de là que l'enseignement secondaire officiel va s'exercer alors en partie sous le signe de l'influence des nouveaux courants philologiques et littéraires.

\section{La place de la culture dans les plans d'études de la IIe République}

3 Tout cela ne va pas être absent du discours didactique de l'époque, comme le montrent les nouveaux plans d'études de la II République qui incluent la littérature dans les études de français. Carmen Roig nous présente ainsi ce nouvel état d'esprit concernant la formation des futurs professeurs de langues étrangères, ce qui coïncide d'ailleurs avec les premières décisions des nouvelles autorités républicaines :

Finalement, le Decreto du 15 septembre 1931 établit à titre d'essai dans les facultés des Lettres de Madrid et Barcelone des épreuves conduisant à une licence en Philologie moderne, option espagnol ou toute autre langue étrangère [...] Les candidats, après avoir suivi trois années d'études en facultés des lettres doivent passer deux séries d'épreuves. La première, commune à toutes les philologies 
modernes, comprend pour moitié les langues classiques et l'arabe et des thèmes de philosophie, histoire et littérature espagnoles. La deuxième série est spécifique aux langues étrangères. Celle de français comprend des exercices écrits et oraux. Parmi les premiers: version latine, version et thème français accompagnés de leur transcription phonétique et deux dissertations, l'une de littérature espagnole, l'autre, en français, de littérature française. Les exercices oraux consistent en des commentaires philologiques d'un texte espagnol et de deux textes français, l'un en français moderne, l'autre en ancien français suivi de deux exposés, l'un sur la littérature espagnole, l'autre sur la française. Tous les exercices concernant soit la langue, soit la littérature française se feront dans cette langue. (Roig 1999 : 243)

C'est dire l'importance qu'on accorde dans les nouveaux plans d'études à la littérature française, y compris celle à laquelle nous nous intéressons ici, la littérature du Moyen Âge, ce qui va avoir bientôt des conséquences dont il faut tenir compte. Les anthologies qui commencent à paraître entre 1931 et 1936 soulignent fortement cette tendance qui s'était déjà amorcée pendant la décennie antérieure, mais qui compte maintenant avec le soutien officiel ${ }^{4}$.

\section{La littérature du Moyen Âge et celle des XVIe et XVII siècles dans le manuel de Jesús Guzmán y Martínez}

5 Un exemple remarquable de la nouvelle situation est l'ouvrage de Jesús Guzmán y Martínez, professeur de langue française dans un lycée de Séville, qui se présente en plus «comme membre $\mathrm{du}$ corps technique d'archivistes, bibliothécaires et archéologues ", ce qui met en évidence une formation universitaire diversifiée. Parmi les différents manuels qu'il publie au long de $1935^{5}$, il y en a un qui attire spécialement notre attention. Le titre est un témoignage indiscutable des nouvelles tendances : $L a$ literatura francesa anterior al siglo XVIII [La littérature française antérieure au XVIII ${ }^{\mathrm{e}}$ siècle] (Madrid 1935) où le Moyen Âge a une place d'exception. Il s'agit d'un livre qui montre d'une façon évidente les intérêts philologiques et littéraires de son auteur, mais dont on pourrait mettre en question l'intérêt pédagogique et didactique, car on ne parvient pas à voir quel était l'usage qu'on pouvait en faire dans les salles de classe, surtout que le livre se présente sans préface. Quoi qu'il en soit, il vaut la peine d'examiner les contenus de ce livre qui est surtout, somme toute, une anthologie littéraire doublée d'une histoire de la littérature française ancienne et classique et où les goûts littéraires de l'auteur semblent avoir pris les devants. L'œuvre est divisée en trois parties : «Edad Media » (Moyen Âge), « Renacimiento » (Renaissance) et «Edad de Oro »(Siècle Classique); chacune des parties contient différents chapitres, tous précédés d'une brève introduction rédigée en espagnol et une adaptation en français moderne des textes présentés. Pour ce qui concerne la partie consacrée au Moyen Âge, sur laquelle nous voulons insister tout spécialement, elle comprend huit chapitres, sans ordre chronologique, où l'auteur tient compte surtout des différents genres littéraires abordés :

Chap. I : Serments de Strasbourg.

Chap. II : Troubadours et trouvères : Arnaud de Marveil ; Bertrand de Born ; Thibault IV.

Chap. III : Charles d'Orléans.

Chap. IV : Villon. 

présente Guzmán, il y a des réussites indiscutables que nous voulons mettre en relief, comme c'est le cas du chapitre III consacré à la poésie de Charles d'Orléans dont il nous présente une ballade et un rondeau. Cela ne peut qu'attirer notre attention, étant donné qu'à l'époque ce prince-poète n'était pas encore un auteur très apprécié en milieu scolaire et universitaire, et cela malgré les importants travaux de Pierre Champion pendant les deux premières décennies du XXe siècle. Charles d'Orléans était encore soumis aux préjugés d'une certaine critique qui l'avait qualifié comme «le dernier poète de la société féodale ». Le choix de Guzmán (1935: 10-11), qui nous présente deux des poèmes les plus représentatifs ${ }^{7}$ du duc d'Orléans, montre donc une connaissance non négligeable de la littérature française du Moyen Âge, ainsi que des goûts littéraires très précis. Et cela malgré des décisions spécialement discutables, comme l'omission de la matière de Bretagne ou les chansons de geste, exception faite de la Chanson de Roland, sans compter certaines erreurs ponctuelles comme la fausse date donnée pour les Serments de Strasbourg (812 au lieu de 842), puis la date de naissance proposée pour Charles d'Orléans (1491 au lieu de 1494) $)^{8}$. On pourrait aussi mettre en question l'opportunité de présenter, dans une anthologie d'auteurs français, des poésies des troubadours dont la langue de création était le provençal'.

C'est à peu près la même présentation pour la littérature du XVIe siècle, divisée dans ce cas en neuf chapitres, où les grands auteurs du XVI $I^{e}$ siècle comme Ronsard, Du Bellay, Rabelais et Montaigne côtoient d'autres auteurs comme les poètes Marot, Mellin de Saint-Gelais, Rémi Belleau, Baif, Desportes, Passerat, Vauquelin de la Fresnaye et Mathurin Régnier ${ }^{10}$ ainsi que l'humaniste Jacques Amyot, traducteur de Plutarque. Quant à la littérature du XVII siècle, que Guzmán appelle «Edad de Oro » - un nom dont on se servait plutôt dans les histoires de la littérature espagnole et qui ne laisse pas de nous surprendre quand on l'applique à la littérature française -, elle est divisée en quinze chapitres, toujours avec une présentation identique à celle des deux premières parties. Tous les grands auteurs classiques y sont présents, mais il n'y manque pas non plus d'autres écrivains comme Guez de Balzac ou Voiture. Sur ce point, Guzmán ne fait que suivre d'autres auteurs d'anthologies scolaires qui l'ont précédé, mais on y décèle néanmoins certaines prises de position que nous tenons à souligner. Nous voulons surtout attirer l'attention sur le nombre de pages consacrées à l'œuvre de Corneille : vingt-six pages (69-95) où les critères de Guzmán au moment de faire son choix se laissent entrevoir. Nous avons affaire à des textes très représentatifs, tirés des pièces « les plus édifiantes » du dramaturge rouennais : Le Cid, Cinna et Polyeucte; des passages que l'auteur de l'anthologie juge appropriés pour des élèves du secondaire, en enchaînant ainsi avec la tradition «morale » venant du XIX ${ }^{\mathrm{e}}$ siècle. Le Cid, dont Guzmán présente plusieurs scènes ${ }^{11}$, était une pièce qui pouvait intéresser des élèves qui en même temps étudiaient ce personnage dans les cours d'histoire de l'Espagne et de littérature espagnole ${ }^{12}$, surtout après la « redécouverte » de ce chevalier castillan grâce aux travaux du médiéviste Menéndez Pidal, parmi lesquels le plus récent était La España del Cid [L'Espagne du Cid], publié en 1929. Puis la tradition scolaire va pousser Guzman à présenter des «modèles moraux » aux usagers de son anthologie, s'intéressant chez 
Racine à Andromaque et à Athalie au détriment de Phèdre, et cela malgré la valeur littéraire de cette pièce. Il y a d'ailleurs un détail sur lequel nous tenons à insister et qui concerne les pages consacrées à Fénelon; car après plus de deux siècles de présence constante des Aventures de Télémaque dans les manuels et anthologies pour l'enseignement du français à l'étranger le manuel de Guzmán laisse la place à d'autres œuvres de l'évêque de Cambrai. Quelles peuvent être les raisons qui le poussèrent à cesser de revisiter le Télémaque, si prisé en milieu scolaire, et à se pencher vers d'autres ouvrages moins connus de Fénelon? Sans doute un désir de renouvellement, car l'heure de ce livre était passée, après avoir été pendant de longues années un repère incontournable pour les enseignants de français. Depuis la seconde moitié du XIX ${ }^{\mathrm{e}}$ siècle l'étoile du Télémaque avait commencé à décliner inexorablement et trois quarts de siècle plus tard les professeurs de français préféraient s'intéresser à d'autres textes de Fénelon, sans doute moins connus, tels ceux consacrés à la thématique religieuse comme c'est le cas du "Sermon pour la fête de l'Épiphanie ${ }^{13}$ " (159-162) ou les Fables (164-166). Mais surtout le goût du Moyen Âge, aussi bien de son histoire que de sa littérature, de la part des professeurs et des auteurs d'anthologies pour l'apprentissage du FLE, réapparaîtra ici : Guzmán se tournera vers les Dialogues des morts de Fénelon, mais il n'y cherchera pas de textes concernant l'Antiquité classique, comme on aurait pu s'y attendre; il préférera nous conduire, encore une fois vers le Moyen Âge, exactement jusqu'au $\mathrm{XV}^{\mathrm{e}}$ siècle pour nous proposer un dialogue entre Louis XI et Philippe de Commines (Guzmán 1935 : 162-164).

\section{Le manuel de Rafael Reyes} entièrement en français exception faite de la préface, met en évidence cet intérêt pour la littérature française ancienne, et surtout sert à souligner encore ce même goût pour le Moyen Âge que l'anthologie de Jesús Guzmán. Il ne s'agit pas dans ce cas d'un simple recueil de textes, où les goûts personnels décident des choix, comme c'est le cas pour l'anthologie de Guzmán, mais d'une histoire de la littérature expliquée à travers les textes, comme le signale son titre: Historia de la literatura francesa ilustrada con textos (Desde los orígenes hasta fines del siglo XVII) [Histoire de la littérature française illustrée par des textes (Depuis les origines jusqu'à la fin du XVII ${ }^{e}$ siècle)], où la littérature médiévale occupe une place majeure. Pour introduire les chapitres consacrés à cette première partie de la littérature française, Reyes présente un premier chapitre où il étudie la formation de la langue, comme on le faisait habituellement dans les histoires de la littérature française du Moyen Âge, puis suivent six chapitres. Le tout est divisé de la façon suivante :

Chap. I : Formation de la langue. Les premiers textes.

Chap. II : La Poésie épique : les chansons de Geste.

Chap. III : Cycle Breton. Cycle Antique.

Chap. IV : La poésie lyrique.

Chap. $\mathrm{V}$ : La poésie satirique, allégorique et didactique.

Chap. VI : Le Théâtre du Moyen Âge.

Chap. VII : La prose au Moyen Âge. Les Chroniqueurs. 

à démêler vraiment toute la complexité de la littérature antérieure au XVI ${ }^{\mathrm{e}}$ siècle. On y voit d'ailleurs une certaine influence des médiévistes espagnols, spécialement de Ramón Ménendez Pidal ${ }^{15}$, et probablement aussi du Manuel illustré d'histoire de la littérature française de Jean Calvet $\left({ }^{8} 1934\right)$, que Rafael Reyes aurait consulté avant l'élaboration de son anthologie. Toute une série de coïncidences concernant les contenus des deux livres, celui de Calvet et celui de Reyes, nous poussent dans cette voi $^{16}$. Les divisions chronologiques et celles qui concernent les genres littéraires son très proches à quelques exceptions près. D'autre part, les pages consacrées à ce que Reyes désigne comme "poésie lyrique " sont quelque peu confuses et embrouillées, sans une distinction nette au moment de fixer les différents apports de cette poésie qui s'étend au cours de cinq ou six siècles; même si certaines approches ne sont pas dépourvues d'intérêt et nous apparaissent plus complètes que celles de Guzmán. La présence des poètes comme Rutebeuf, Eustache Deschamps et Christine de Pisan - à côté de Charles d'Orléans et Villon - doit attirer forcément notre attention. Les mots que Reyes dédie en guise d'introduction à Christine de Pisan ${ }^{17}$ montrent un certain choix personnel qui est bien loin de la position critique de Gustave Lanson lui-même. Celui-ci montre une sévérité extrême à l'égard de l'écrivaine, une position critique à laquelle le professeur espagnol ne semble pas avoir prêté la moindre attention au cas où il eût consulté le manuel de l'auteur français :

Ne nous arrêtons pas à l'excellente Christine de Pisan, bonne fille, bonne épouse, bonne mère, du reste un des plus authentiques bas-bleus qu'il y ait dans notre littérature, la première de cette insupportable lignée de femmes auteurs, à qui nul ouvrage sur aucun sujet ne coûte, et qui pendant toute la vie que Dieu leur prête, n'ont affaire que de multiplier les preuves de leur infatigable facilité, égale à leur universelle médiocrité. (Lanson 1970 [1894] : 167)

Au-delà des influences et des sources, Rafael Reyes a su adapter son Histoire de la littérature française aux prescriptions des nouveaux plans d'études des années de la $\mathrm{II}^{\mathrm{e}}$ République où la connaissance pratique de la langue, grâce à la compréhension et à l'expression orale et écrite, se combine avec la connaissance de la «civilisation française ». Et là, la littérature, moyennant la lecture et éventuellement la traduction, devait faire l'objet d'une considération et d'un intérêt spéciaux (Fernández Fraile et Suso 1999 : 294-295). C'est un programme ambitieux et quelque peu contradictoire où la dimension communicative s'entrecroise avec des aspects éminemment culturels et littéraires. Reyes semble avoir su saisir l'esprit des législateurs de l'époque :

Notre Littérature française [...] répond tout à fait aux prescriptions du Programme Officiel pour les élèves de la troisième année du nouveau plan de l'enseignement secondaire et elle sera suivie d'un deuxième volume qui comprendra depuis le XVIII siècle jusqu'aux auteurs de nos jours, adapté donc aussi aux directives de ce même Programme officiel pour les élèves de la quatrième année des études secondaires actuelles. De cette façon nous avons respecté non seulement les prescriptions légales du nouveau plan du Secondaire mais aussi le principe suivi par la didactique universelle qui suggère l'importance de mettre en avant les atouts de l'Histoire de la Littérature d'une langue moyennant les textes et l'analyse des ceux-ci, ce que les anciens manuels laissaient de côté car ils souffraient du grave défaut de donner à propos des auteurs, les ouvrages qu'ils ont écrits et la critique qui s'en détache une connaissance tellement abstraite de la Littérature qu'il n'était pas possible que les élèves pussent s'en servir plus tard pour faire des compositions littéraires, car il leur manquait les bons modèles qu'ils méconnaissaient pratiquement. Et si ceci est intéressant et même nécessaire pour ceux qui étudient la littérature de leur propre 
langue, il faut penser son importance pour celui qui étudie une langue étrangère.

(Reyes $1935: 5-6)$ [ma traduction]

Reyes semble donc remettre en question l'habitude de présenter l'histoire de la littérature à travers la vie des auteurs et la liste de leurs œuvres, sans donner aux textes l'attention qu'ils méritent vraiment. C'est sans doute pour cette raison que quand il présente les textes médiévaux, pour faciliter leur lecture et leur compréhension il propose la traduction correspondante en français moderne, mais sans délaisser pour autant les textes originaux que l'élève doit, selon lui, connaître de première main. Ce qui l'intéresse finalement c'est que l'élève puisse se familiariser avec les textes et en tirer profit pour l'acquisition de la langue étrangère. Il a le même souci pour les autres parties de la littérature française antérieure au XIX ${ }^{\mathrm{e}}$ siècle, même si dans ce cas les intérêts ne sont pas identiques, car au fur et à mesure qu'on avance dans le temps les problèmes de compréhension linguistique doivent forcément se réduire. On y voit toujours le souci d'établir un certain parallélisme entre la littérature française et la littérature espagnole, une approche comparative qui pourrait aider les élèves au moment de se mesurer à certaines réalités littéraires. Somme toute, le livre de Rafael Reyes, comme cela serait aussi le cas pour celui de Jesús Guzmán y Martínez, semble tenir compte du fait que bien des élèves, qui se servaient de ces livres pour apprendre le français, étudiaient en même temps la littérature espagnole, y compris celle du Moyen Âge, à laquelle pour des raisons d'opportunité historique on accordait alors une importance indiscutable, comme nous venons de le voir.

\section{En guise de conclusion : le Moyen Âge dans les manuels de français après la Guerre civile}

Les manuels de français postérieurs à la Guerre civile ne renoncent pas à la présence du Moyen Âge dans leurs pages. Mais cette présence ne peut pas s'expliquer de façon identique à celle des premières décennies $\mathrm{du} \mathrm{XX}^{\mathrm{e}}$ siècle et tout spécialement à celle $\mathrm{du}$ temps de la Seconde République. Les raisons de cette nouvelle présence du Moyen Âge à partir de 1940 s'expliquent surtout pour des raisons idéologiques et religieuses. Le Moyen Âge aurait été vu par les nouveaux responsables de l'éducation de l'Espagne franquiste comme une époque très favorable pour les valeurs chrétiennes qu'il fallait transmettre aux élèves. C'est pour cette raison que le Moyen Âge continue à être présent dans les manuels qui paraissent entre 1940 et $1970^{18}$, spécialement dans la cinquième année du secondaire où il était obligatoire pour les élèves de français de travailler sur des textes littéraires, y compris des textes littéraires médiévaux ${ }^{19}$. Mais il s'agissait surtout d'avoir recours à des textes d'inspiration essentiellement chrétienne dont on pouvait tirer des leçons "morales » et "patriotiques", d'accord avec les postulats franquistes. Une situation qui se maintiendra jusqu'en 1970, lorsque la nouvelle loi d'éducation des dernières années du franquisme supprimera la littérature dans les manuels de français, au moment où les nouvelles méthodes audio-visuelles pour l'enseignement des langues étrangères commencent à s'imposer. 


\section{BIBLIOGRAPHIE}

Sources primaires

CALVET, Jean $\left({ }^{20} 1961\right.$ [ $\left.\left.{ }^{8} 1934\right]\right)$. Manuel illustré d'histoire de la Littérature Française. Paris : Éditions De Gigord.

(1961 [1934]). Morceaux choisis des auteurs français. Paris : Éditions De Gigord.

GALICIA AYALA, Juan. (1898). Le Gaulois. Método completo para la enseñanza de la lengua francesa. Port Mahon : Impr. B. Fàbregues.

GUZMÁN Y MARTínEZ, Jesús. (1935). La literatura francesa anterior al siglo XVIII. Madrid : Imprenta Martosa.

LANSON, Gustave (1970 [1894]). Histoire de la littérature française. Paris : Hachette.

NISARD, Désiré (1844-1864). Histoire de la littérature française, Paris : Firmin Didot, 1844-1864, 4 tomes.

REYES, Rafael (1935). Historia de la literatura francesa ilustrada con textos (Desde los orígenes hasta fines del siglo XVII), t. I. Madrid : Nuevas Gráficas.

Sources secondaires

Amalvi, Christian (1998). Le goût du Moyen Âge. Paris : Plon.

BRUÑA CUEVAS, Manuel (2016). « Rafael Reyes et ses dictionnaires français-espagnol et espagnolfrançais (1926-1928) », Documents pour l'histoire du français langue étrangère ou seconde, 56, 189-206.

DEYERMOND, Alan (1979). Edad Media. In Francisco Rico (dir.), Historia crítica de la literatura española, vol. 1. Barcelone : Crítica.

FISCHER, Denise, GARCíA BASCUÑANA, Juan F. \& GóMEZ, Maria Trinidad (2004). Repertorio de gramáticas y manuales para la enseñanza del francés en España (1565-1940). Barcelone : PPU.

GARCíA BASCUÑANA, Juan F. (2016). « Guzmán y Martínez, Jesús » et « Reyes, Rafael ». In Juan F. García Bascuñana (dir.), Diccionario de historia de la enseñanza del francés en España (siglos XVI-XX). Tarragone : URV. En ligne :

[http://www.grelinap.recerca.urv.cat/projectes/diccionario-historia-ensenanza-francesespana/] (26 septembre 2018).

GARCÍA BASCUÑANA, Juan \& SANTOS, Ana Clara (2012). « Le texte littéraire dans l'enseignement du FLE : histoire, variations et perspectives ", Histoire internationale de l'enseignement du français langue étrangère ou seconde; problèmes, bilans et perspectives. Le français dans le monde, Recherches et applications. 52, 120-130.

MENÉNDEZ PIDAL, Ramón (1979) : « Los juglares y los orígenes de la literatura española ». In A. Deyermond (dir.), Edad Media, vol 1, 15-19. Barcelone : Crítica.

PiqueR DesvauX, Alicia (2016). « Antologías literarias ». In Juan F. García Bascuñana (ed.), Diccionario de historia de la enseñanza del francés en España (siglos XVI-XX). Tarragone : URV. En ligne : [http://www.grelinap.recerca.urv.cat/projectes/diccionario-historia-ensenanza-francesespana/] (26 septembre 2018). 
RoIG, Carmen. (1999). « Le débat méthodologique sur l'enseignement des langues étrangères dans les pages du Boletín de l'Institución Libre de Enseñanza (BILE) », Documents pour l'histoire du français langue étrangère ou seconde, 23, 227-247.

\section{NOTES}

1. Nous citons expressément le titre du livre de Christian Amalvi (1998).

2. L'une des exceptions que nous avons trouvée au XIXe siècle est un manuel de 1898 d'un professeur de lycée, Juan Galicia Ayala, Le Gaulois. Método completo para la enseñanza de la lengua francesa [Le Gaulois. Méthode complète pour l'enseignement de la langue française (trad.)]. Nous y trouvons deux textes médiévaux: Les Serments de Strasbourg et un autre tiré de la Chanson de Roland présentant la mort de celui-ci.

3. Tentative pédagogique réalisée en Espagne dans les dernières décennies du $\mathrm{XIX}^{\mathrm{e}}$ siècle et les premières du XX' $\mathrm{XX}^{\mathrm{e}}$, qui s'inspirait de la philosophie de Karl Christian Friedrich Krause (Krausisme) et qui comme force novatrice eut une grande influence dans certains milieux progressistes.

4. Cf. l'entrée « Antologías literarias » (Piquer Desvaux 2016).

5. Guzmán y Martínez publie six manuels pour l'enseignement du FLE, tous pendant l'année 1935, bien que quelques-uns de ces livres soient seulement des réimpressions ou des rééditions d'éditions antérieures, quelques-unes des années 1920. Il convient de souligner que la plupart contiennent des textes littéraires antérieurs au $\mathrm{XX}^{\mathrm{e}}$ siècle, ce qui montre certaines affinités culturelles et littéraires du professeur sévillan. Cf. Fischer, García Bascuñana \& Gómez, M.T. (2004 : 187-190) et l'entrée « Guzmán y Martínez, Jesús » (García Bascuñana 2016).

6. C'est ce qu'avait affirmé, au XIX ${ }^{\mathrm{e}}$ siècle, l'historien de la littérature Désiré Nisard (1844-1864 : t. 1,202$)$ qui avait complété ses préjugés à l'égard de Charles d'Orléans avec des mots qui malgré leur intentionnalité négative peuvent être pris à son insu comme un éloge, au moins dans sa première partie : «Il empruntait à Jean de Meung ses allégories et à Pétrarque ses idées, voilà pourquoi, il est si rare d'y trouver un accent vrai et une expression forte ».

7. Il s'agit d'une ballade (« N'a pas longtemps qu'allai parler ») de la première époque de Charles d'Orléans, probablement antérieure à son emprisonnement en Angleterre après la débâcle d'Azincourt, et où l'influence de la lyrique courtoise traditionnelle est évidente ; puis d'un de ses rondeaux les plus connus ( «e temps a laissé son manteau ») et l'un des plus appréciés en milieu scolaire, qui appartient tout probablement aux premières années du retour à Blois après son long exil (ballade 6 et rondeau 31 d'après l'édition de Pierre Champion, Poésies, 2 vol. , Paris, Honoré

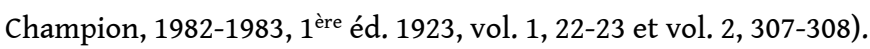

8. Ce qui ne laisse pas de nous surprendre, puisque depuis la parution de la Vie de Charles d'Orléans de Pierre Champion en 1911 (rééd. 2010), la date de naissance de Charles d'Orléans était bien établie (P. Champion, Vie de Charles d'Orléans, p. 1, cf. ci-dessous fin note 20).

9. Guzmán fait un choix de poèmes de Bertrand de Born et d'Arnaut de Maruelh, qu'il présente sous le nom francisé d'Arnaud de Marveil.

10. Mais, on ne sait pas exactement pour quelle raison il oublie un poète aussi remarquable qu'Agrippa d'Aubigné.

11. Dix-neuf des vingt-six pages consacrées à Corneille présentent différentes scènes du Cid.

12. Certains professeurs pouvaient en profiter pour faire une approche comparative entre la pièce de Corneille et Las mocedades del Cid (La jeunesse du Cid) de l'auteur dramatique espagnol Guillén de Castro, qui avait été probablement la source de Corneille.

13. Guzmán aurait pu connaître ce texte religieux de Fénelon, à peine présent dans les anthologies scolaires françaises de son temps, grâce à la huitième édition de l'Histoire de la 
littérature française (manuel et morceaux choisis) de Jean Calvet, qui venait d'être publiée un an avant le livre de Guzmán (Calvet 1961 [1934] : 427-428).

14. À propos de Rafael Reyes, nous renvoyons à l'article de Manuel Bruña, « Rafael Reyes et ses dictionnaires français-espagnol et espagnol-français (1926-1928)»(2016: 189-206). Cf. aussi l'entrée « Reyes, Rafael » (García Bascuñana 2016).

15. Menéndez Pidal avait publié pendant les deux premières décennies du $\mathrm{XX}^{\mathrm{e}}$ siècle toute une série d'œuvres se rapportant à la littérature espagnole du Moyen Âge, qui ont été fondamentales pour les études médiévales: La epopeya castellana a través de la literatura española [L'épopée castillane à travers la littérature espagnole] (1910); Cantar del mío Cid: texto, gramática y vocabulario [Chanson du Moi Cid : texte, grammaire et vocabulaire] (1908-1912); Poesía juglaresca y juglares [La poésie épique et les jongleurs] (1924) ; Flor nueva de romances viejos_[Nouvelle fleur des vieilles romances] (1928) ; La España del Cid [L'Espagne du Cid] (1929). Cf. Deyermond (1979: 15-19).

16. Aussi bien Rafael Reyes que Jesús Guzmán auraient pu connaître aussi d'autres histoires de la littérature française publiées en France comme, par exemple, l'incontournable Histoire de la littérature française de Gustave Lanson. Certains chapitres consacrés à la littérature du Moyen Âge ainsi que d'autres concernant les XVI ${ }^{e}$ et XVII ${ }^{\mathrm{e}}$ siècles laissent entrevoir cette possibilité. À titre d'exemple comparons le traitement de la matière de Bretagne dans le livre de Lanson (1970 - $1^{\text {ère }}$ éd. 1894 : 46-64) et dans celui de Reyes (1935 : 25-34) où il y a de nombreuses coïncidences.

17. Reyes dit à propos de Christine de Pisan qu'elle « écrivit d'abord pour se consoler, puis pour vivre. Son œuvre poétique est considérable» (1935:38).

18. L'approbation d'une nouvelle loi d'éducation (Ley General de Educación) en 1970 est venue changer l'enseignement espagnol et introduire de nouveaux plans d'études qui modifient la donne de l'enseignement des langues étrangères et tout particulièrement du français ( $c f$. García Bascuñana et Santos, 2012).

19. «La Littérature française par les textes » était le nom de la matière qui était étudiée pendant cette cinquième année de français.

\section{RÉSUMÉS}

Cette étude aborde un aspect négligé en Espagne par ceux qui se sont intéressés à la présence de la littérature dans l'enseignement du français. Ce qui a été justifié avec des arguments divers. Pas même le "goût du Moyen Âge " qui s'impose en France au XIX ${ }^{\mathrm{e}}$ siècle n'aurait favorisé sa présence dans les manuels de français. Pratiquement aucun auteur de manuels ou d'anthologies littéraires du XIX ${ }^{\mathrm{e}}$ siècle n'a inclus de textes littéraires médiévaux dans ses œuvres. Mais cette situation commence à changer dans les années 1920-1930 où la "redécouverte » espagnole du Moyen Âge a fait naître l'intérêt pour cette période. Une bonne partie des auteurs de manuels et des professeurs de français de l'époque seront avant tout des romanistes, avec toutes les conséquences pratiques que cela implique. Cette situation va perdurer d'ailleurs après la fin de la Guerre civile grâce à un intérêt renouvelé pour le Moyen Âge, suscité alors surtout pour des raisons purement idéologiques.

This study delves into a Spanish topic underestimated by all those interested in the presence of literature in the teaching of French, which has been justified on diverse grounds. Not even the «taste for the Middle Ages» that thrived throughout the XIX century would have favoured the 
presence of medieval literature in French manuals. Practically, no nineteenth-century manual or literary anthology authors include medieval literary texts in their works. However, this situation begins to change in the 1920s and 1930s when the Spanish "rediscovery " of the Middle Ages gave place to a renewed interest in that period. A good deal of French manuals and teachers at that time are, above all, Romanists, with all the practical consequences that would entail. This situation will endure throughout the end of the Civil War in 1939 thanks to an increased interest in the Middle Ages raised then, mainly, by sheer ideological reasons.

\section{INDEX}

Keywords : teaching of French as a FL, French literature, literary texts, Middle Ages, manuals, study plans, Spain

Mots-clés : enseignement du FLE, littérature française, textes littéraires, Moyen Âge, manuels, plans d'études, Espagne

\section{AUTEUR}

\section{JUAN FRANCISCO GARCÍA BASCUÑANA}

Universitat Rovira i Virgilijuanfrancisco.garcia@urv.cat 